\title{
Prevalence and Characteristics of Symptomatic Pneumonitis After Radiotherapy of Patients With Locally Advanced Lung Cancer
}

\author{
DIRK RADES ${ }^{1}$, ESTHER GLATZEL $^{1}$, ELISA M. WERNER $^{1}$ and SABINE BOHNET ${ }^{2}$ \\ ${ }^{1}$ Department of Radiation Oncology, University of Lübeck, Lübeck, Germany; \\ ${ }^{2}$ Department of Pulmonology, University of Lübeck, Lübeck, Germany
}

\begin{abstract}
Background/Aim: Radiotherapy of locally advanced lung cancer often requires high doses potentially leading to pneumonitis. This study evaluated the rate of symptomatic pneumonitis and characteristics in these patients. Patients and Methods: This study included 278 patients irradiated for locally advanced lung cancer between 2016 and 2019. In patients experiencing symptomatic pneumonitis, patient and treatment characteristics were analyzed. Results: Pneumonitis was diagnosed in 21 patients (7.6\%) after a median of 9 (123) weeks. Ipsilateral lungs received mean doses $>13$ Gy in 21 (100\%) and $>20$ Gy in 15 patients $(71.4 \%)$. Seventeen patients $(81.0 \%)$ received chemotherapy and/or immunotherapy, 12 $(57.1 \%)$ had significant cardiovascular disease (all 21 patients had risk factors), 11 (52.4\%) were heavy smokers ( $\geq 40$ pack years), 7 (33.3\%) were aged $\geq 74$ years, 5 (23.8\%) had chronic inflammatory disease and $4(19.0 \%)$ had previous tumors. Conclusion: Overall pneumonitis rate was $7.6 \%$. Frequent characteristics included high mean lung doses, systemic treatment, cardiovascular disease (and risk factors), heavy smoking, older age, chronic inflammatory disease and history of a previous tumor.
\end{abstract}

Lung cancer is the second most common solid cancer in Europe and Northern America (1). The majority of patients with small-cell lung cancer (SCLC) receive radiotherapy in combination with chemotherapy. Also, many patients with locally advanced non-small-cell lung cancer (NSCLC) receive radiotherapy, if they are not candidates for surgery. Major goals of radiotherapy include local disease control and

Correspondence to: Prof. Dirk Rades, MD, Department of Radiation Oncology, University of Lübeck, Lübeck, Ratzeburger Allee 160, 23562 Lübeck, Germany. Tel: +49 45150045401, Fax: +49 45150045404, e-mail: dirk.rades@uksh.de

Key Words: Lung cancer, advanced disease, radiotherapy, symptomatic pneumonitis, prevalence, characteristics. improvement of the patients' overall prognoses (2). In the majority of cases, the treatment approach is curative. Therefore, high radiation doses are often required that may lead to pneumonitis. Pneumonitis can occur up to 21 weeks following radiotherapy (3). Radiation pneumonitis must be considered a serious complication that was reported to be fatal in approximately $2 \%$ of the patients (2). Therefore, it would be desirable to identify patients, who are at high risk of developing radiation pneumonitis, prior to the start of treatment. These patients would require a closer monitoring during the course of radiotherapy and several months following treatment. Moreover, in the literature, the prevalence of symptomatic pneumonitis after irradiation of lung cancer varies considerably (4-7). Thus, more studies are required to properly identify the rate of symptomatic pneumonitis, particularly studies using modern precision radiotherapy techniques. Therefore, this study was performed aiming to identify the prevalence of pneumonitis and potential risk factors for this complication in patients with locally advanced lung cancer irradiated with volumemodulated arc therapy (VMAT).

\section{Patients and Methods}

In this retrospective study, which was approved by the ethics committee of the University of Lübeck, 278 patients irradiated for locally advanced lung cancer between 2016 and 2019 were evaluated with respect to the development of symptomatic (grade $\geq 2$ ) pneumonitis. Patients with locally advanced disease who had distant metastases were also included. Radiotherapy was performed as modern precision therapy, i.e. VMAT. Patients received conventionally fractionated radiotherapy of $5 \times 2$ Gy per week (on 5 consecutive days).

In these patients developing pneumonitis following radiotherapy, clinical and treatment characteristics were analyzed to detect potential risk factors for this treatment-related complication. Clinical characteristics included age, gender, histology, tumor stage, tumor site, co-morbidity, history of smoking and history of another tumor. Treatment characteristics included mean dose to the ipsilateral lung and type of systemic treatment. In addition, time to progression 
(local and/or distant failure) was evaluated in the patients developing pneumonitis, which was calculated from the end of radiotherapy.

\section{Results}

In the entire cohort of 278 patients, pneumonitis was diagnosed in 21 patients $(7.6 \%)$ after a median of 9 weeks (range $=1-23$ weeks) following irradiation. Leading symptoms were dyspnea in 18 patients $(85.7 \%)$ and cough in 12 patients (57.1\%). All patients received prednisolone for up to 13 weeks. Pneumonitis resolved in 18 patients $(85.7 \%)$. In one patient, symptoms had already improved but became worse after prednisolone was stopped. A second course of prednisolone was administered, and finally pneumonitis resolved. In one patient, dyspnea and cough were persistent for 1 year, before they markedly improved. Also, one patient was lost to follow up soon after initiation of the prednisolone treatment.

Of the 21 patients, 4 (19.0\%) had SCLC, 9 (42.9\%) adenocarcinoma (AC) and 8 patients $(38.1 \%)$ squamous cell carcinoma (SCC). The median total radiation dose was 60 Gy in the entire cohort, as well as in all three histologic subgroups. Mean doses to the ipsilateral lung were $>13 \mathrm{~Gy}$ in 21 patients $(100 \%),>20$ Gy in 15 patients $(71.4 \%)$ and $>27$ Gy in five patients (23.8\%). According to Quantitative Analyses of Normal Tissue Effects in the Clinic (QUANTEC), these doses are associated with a risk of $10 \%$, $20 \%$ and $40 \%$, respectively, to develop symptomatic pneumonitis (8).

Radiotherapy was combined with systemic treatment in 17 patients $(81.0 \%)$. The type of systemic treatment depended on the histology of the primary tumor. Of the patients with SCLC, three patients received 4 courses of cisplatin/etoposide ( 2 courses concurrently with radiotherapy) and one patient 2 courses of carboplatin/etoposide concurrently with radiotherapy. Of the patients with $\mathrm{AC}$, three patients received 2-4 courses of cisplatin/pemetrexed (2 courses concurrently with radiotherapy in 2 patients), one patient 6 weekly applications of paclitaxel during radiotherapy, one patient afatinib over 6 months prior to radiotherapy and one patient durvalumab over 2 months as consolidation therapy after radiotherapy. Three patients with AC did not receive systemic treatment. In the group of patients with SCC, two patients received 4 courses of cisplatin/vinorelbine (2 courses concurrently with radiotherapy). One patient additionally received durvalumab consolidation therapy, two patients 6 weekly applications of paclitaxel during radiation, one patient 6 weekly applications of vinorelbine, one patient 2 courses of carboplatin/nab-paclitaxel and one patient pembrolizumab over 2 months after radiation treatment. One patient with SCC did not receive systemic treatment.

With respect to the patients characteristics (Table I), it should be noted that 12 patients $(57.1 \%)$ had significant cardiovascular disease (all 21 patients had corresponding risk factors including diabetes mellitus, hypertension and/or history of smoking), 11 patients (52.4\%) were heavy smokers (defined as $\geq 40$ pack years), seven patients (33.3\%) were aged $\geq 74$ years and 12 patients $(57.1 \%)>65$ years, five patients $(23.8 \%)$ had chronic inflammatory disease (four patients with bronchial asthma and one with rheumatoid arthritis) and four patients (19.0\%) had a history of a previous tumor (two patients with larynx cancer, one with breast cancer and one with bilateral adrenal incidentaloma).

In the 18 patients with a follow up of at least 6 months, progression of lung cancer occurred in 15 patients $(83.3 \%)$ after a median of 9 months (range=3-17 months) following radiotherapy. Five patients $(27.8 \%)$ experienced progression within 6 months and 12 patients (66.7\%) within 12 months. Sites of first failure were local progression alone in one patient $(5.6 \%)$, distant progression alone in 12 patients (66.7\%) and both in two patients $(11.1 \%)$. In the group of patients with SCLC, progression occurred in all four patients $(100 \%)$ after a median of 8 months (range $=3-11$ months). Of the nine patients with $\mathrm{AC}$, six patients (66.7\%) had progression of their disease after a median of 10.5 months (range $=3-17$ months) months, and all five evaluable (appropriate length of follow up) patients with SCC (100\%) had progression after a median of 7 months (range $=6-17$ months).

\section{Discussion}

Radiotherapy alone or in combination with systemic therapies is commonly used for treating locally advanced lung cancers (2). As the prognosis of these patients is often limited, considerable research is carried out to improve their outcomes (9-13). If radiotherapy is administered, high doses are often required, particularly if the treatment approach is considered curative. These radiation doses may be associated with pneumonitis that can be severe and, in a very small proportion of patients, even fatal (2).

Therefore, it would be important to identify patients, who are at high risk of experiencing radiation pneumonitis, before starting the treatment to adjust the dose-fractionation regimen and provide close monitoring of the patients during and after radiotherapy. The present study aimed to contribute to the identification of potential risk factors for this complication in patients irradiated for locally advanced lung cancer. In the present cohort, the prevalence of pneumonitis following radiotherapy was lower than in previous studies (4-7). This finding may be explained by the facts that in the present study radiotherapy was performed with a highprecision technique (VMAT), that the patients were monitored closely during radiation treatment, which led to premature termination of the radiation treatment in case of severe symptoms, complications or significant deterioration 
Table I. Patient characteristics of the 21 patients with pneumonitis.

\begin{tabular}{lccccccc}
\hline $\begin{array}{l}\text { Patient } \\
\text { number }\end{array}$ & $\begin{array}{c}\text { Age } \\
\text { (years) }\end{array}$ & Gender & $\begin{array}{c}\text { Tumor } \\
\text { site }\end{array}$ & Histology & $\begin{array}{c}\text { TNM } \\
\text { stage }\end{array}$ & $\begin{array}{c}\text { UICC } \\
\text { stage }\end{array}$ & $\begin{array}{c}\text { Cardiovascular disease } \\
\text { and risk factors }\end{array}$ \\
\hline 1 & 59 & Male & LL & SCLC & T1 N3 M0 & LD & CHD, DM, HT, AS \\
2 & 54 & Female & UL & AC & T2 N3 M0 & IIIB & T0py \\
3 & 68 & Female & LL & AC & T2 N2 M0 & IIIA & CS, TIA, 40py \\
4 & 74 & Female & LL & SCC & T3 N3 M0 & IIIC & CHD, HT, PAD, >50py \\
5 & 56 & Female & UL & SCLC & T4 N3 M0 & LD & CS, HT, 35py \\
6 & 74 & Male & Central & AC & T4 N3 M1a & IVA & CHD, HT, 30py \\
7 & 53 & Female & UL & AC & T2 N3 M0 & IIIB & T0py \\
8 & 87 & Male & UL & AC & T2 N3 M1a & IVA & CHD, MI, HT \\
9 & 61 & Male & Central & SCLC & T4 N3 M1 & ED & HT, 40py \\
10 & 46 & Male & Central & AC & T4 N3 M1c & IVB & 20py \\
11 & 82 & Female & ML & SCC & T4 N2 M0 & IIIB & CHD, HT \\
12 & 78 & Male & LL & SCC & T4 N2 M1c & IVB & CHD, CMP, HT, 50py \\
13 & 69 & Female & UL & SCC & T4 N2 M0 & IIIB & DM, HT \\
14 & 88 & Male & UL & AC & T2 N3 M0 & IIIB & CS, apoplexy, HT, >50py \\
15 & 68 & Male & UL & SCLC & T1 N2 M0 & LD & L0py \\
16 & 68 & Female & UL & AC & T1 N3 M0 & IIIB & Apoplexy, HT, 35py \\
17 & 62 & Female & UL & SCC & T4 N2 M1b & IVB & HT \\
18 & 59 & Male & UL & SCC & T3 N3 M0 & IIIC & DM, 40py \\
19 & 75 & Male & Central & SCC & T4 N2 M0 & IIIB & HT, AS, 50py \\
20 & 68 & Male & LL & AC & T3 N3 M1c & IVB & Apoplexy, HT, 45py \\
21 & 58 & Male & UL & SCC & T3 N2 M0 & IIIB & HT, 60py \\
\hline
\end{tabular}

LL: Lower lobe; UL: upper lobe; ML: middle lobe; SCLC: small-cell lung cancer; AC: adenocarcinoma, SCC: squamous cell carcinoma; UICC: Union for International Cancer Control; LD: limited disease; ED: extensive disease; CHD: coronary heart disease; DM: diabetes mellitus; HT: hypertension; AS: arteriosclerosis; py: pack years; CS: carotid stenosis; TIA: transient ischemic attack; PAD: peripheral arterial disease; MI: myocardial infarction; CMP: cardiomyopathy.

of the patient's performance score, and that there has been a very close collaboration with the department of pulmonology. Another important reason may be the design of the study. Since the data were obtained retrospectively from patient files, radiation pneumonitis may have been missed in some patients following radiotherapy.

The main goal of the present study was the identification of potential risk factors for symptomatic pneumonitis. We found that in patients experiencing radiation pneumonitis, some characteristics were comparatively common. These characteristics included high mean radiation doses to the ipsilateral lung, administration of chemotherapy and/or immunotherapy during or close to radiotherapy, cardiovascular disease or corresponding risk factors, history of heavy smoking ( $\geq 40$ pack years), older age ( $\geq 74$ years), history of chronic inflammatory disease and history of a previous tumor. Some of these characteristics have been previously described to be associated with pneumonitis after irradiation of lung cancer. A pooled analysis of 88 studies identified the mean lung dose as a significant $(p=0.027)$ risk factor for radiation pneumonitis after stereotactic radiation therapy of thoracic tumors (14). Moreover, according to QUANTEC, mean lung doses can be correlated with the risk of symptomatic pneumonitis (8). The fact that chemotherapy and immunotherapy can increase the risk of radiation pneumonitis has been suggested in several studies (15-18). This association can be explained by the fact that these agents have a radio-sensitizing effect that also affects normal tissues.

The risk factors of cardiovascular disease, diabetes mellitus and hypertension, have been described by several authors to be potential risk factors for radiation pneumonitis (18-21). The prognostic role of pre-treatment cardiac comorbidity has been investigated only in one retrospective study (22). In that study, 259 lung cancer patients were included, who received definitive radiotherapy or radiochemotherapy. Of 75 patients with pre-treatment cardiac comorbidity, 33 patients $(44.0 \%)$ developed radiation-induced lung toxicity (odds ratio $=2.58, p<0.001$ ). This association may be explained by the reduced blood flow due to arteriosclerosis, which may impair defense mechanisms necessary to prevent pneumonitis. Smoking has also been previously identified as a risk factor for pneumonitis (18, 23 ). In the study of Li et al., $\geq 40$ pack years, the cut-off used for the present study, were significantly $(p=0.012)$ associated with grade $\geq 3$ radiation pneumonitis (23). Older age is another previously described risk factor (14-16). In our study, more than half of the patients were $>65$ years of age 
and one third $\geq 74$ years, which supports the findings of the previous studies.

In addition to these characteristics, history of chronic inflammatory disease and history of another tumor were present in our cohort more frequent than expected. Potential associations of these two characteristics with radiation pneumonitis were found also in our previous study investigating pneumonitis following irradiation of breast cancer (24). Bronchial asthma is a chronic inflammatory lung disease, and rheumatoid arthritis can also be associated with pulmonary involvement $(25,26)$. The history of another tumor may be explained by a reduced DNA repair capacity, which has been described for several tumor types including lung cancer and can result in increased radio-sensitivity and radiation-related toxicity (27-29). The idea that patients developing radiation pneumonitis may have a reduced DNA repair capacity is further supported by the fact that the patients in our cohort had a poor prognosis in terms of progression-free survival.

In conclusion, the overall pneumonitis rate was comparatively low. Characteristics potentially associated with radiation pneumonitis included high mean lung doses, systemic treatment, cardiovascular disease (and corresponding risk factors), heavy smoking, older age, history of chronic inflammatory disease and history of another tumor.

\section{Conflicts of Interest}

On behalf of all Authors, the corresponding Author states that there is no conflict of interest related to this study.

\section{Acknowledgements}

As part of the project NorDigHealth, this study was funded by the European Regional Development Fund through the Interreg Deutschland-Danmark program.

\section{Authors' Contributions}

D.R., E.G., E.M.W. and S.B. participated in the design of the study. D.R., E.G., E.M.W. and S.B. provided data and performed analyses and interpretation of the data. D.R. drafted the manuscript, which was reviewed and approved in its final form by all Authors.

\section{References}

1 Siegel RL, Miller KD and Jemal A: Cancer statistics, 2019. CA Cancer J Clin 69: 7-34, 2019. PMID: 30620402. DOI: 10.3322/ caac. 21551

2 Verma V, Simone CB 2nd and Werner-Wasik M: Acute and late toxicities of concurrent chemoradiotherapy for locally-advanced non-small cell lung cancer. Cancers (Basel) 9: pii:E120, 2017. PMID: 28885561. DOI: 10.3390/cancers9090120

3 Vasiljevic D, Arnold C, Neuman D, Fink K, Popovscaia M, Kvitsaridze I, Nevinny-Stickel M, Glatzer M, Lukas P and Seppi
T: Occurrence of pneumonitis following radiotherapy of breast cancer - A prospective study. Strahlenther Onkol 194: 520-532, 2018. PMID: 29450591. DOI: 10.1007/s00066-017-1257-z

4 Dang J, Li G, Lu X, Yao L, Zhang S and Yu Z: Analysis of related factors associated with radiation pneumonitis in patients with locally advanced non-small-cell lung cancer treated with three-dimensional conformal radiotherapy. J Cancer Res Clin Oncol 136: 1169-1178, 2010. PMID: 20130912. DOI: 10.1007/s00432-010-0764-4

5 Inoue A, Kunitoh H, Sekine I, Sumi M, Tokuuye K and Saijo N: Radiation pneumonitis in lung cancer patients: a retrospective study of risk factors and the long-term prognosis. Int J Radiat Oncol Biol Phys 49: 649-655, 2001. PMID: 11172945. DOI: 10.1016/s0360-3016(00)00783-5

6 Hegi F, D’Souza M, Azzi M and De Ruysscher D: Comparing the outcomes of stereotactic ablative radiotherapy and nonstereotactic ablative radiotherapy definitive radiotherapy approaches to thoracic malignancy: A systematic review and meta-analysis. Clin Lung Cancer 19: 199-212, 2018. PMID: 29370978. DOI: 10.1016/j.cllc.2017.11.006

7 Zhou Z, Song X, Wu A, Liu H, Wu H, Wu Q, Liu Y, Li Y, Cai $\mathrm{Y}$ and Liang S: Pulmonary emphysema is a risk factor for radiation pneumonitis in NSCLC patients with squamous cell carcinoma after thoracic radiation therapy. Sci Rep 7: 2748, 2017. PMID: 28584268. DOI: 10.1038/s41598-017-02739-4

8 Bentzen SM, Constine LS, Deasy JO, Eisbruch A, Jackson A, Marks LB, Ten Haken RK and Yorke ED: Quantitative analyses of normal tissue effects in the clinic (QUANTEC): an introduction to the scientific issues. Int J Radiat Oncol Biol Phys 76(3 Suppl): S3-9, 2010. PMID: 20171515. DOI: 10.1016/j.ijrobp.2009.09.040

9 Wada K, Kishi N, Kanayama N, Hirata T, Morimoto M, Konishi $\mathrm{K}$, Imamura $\mathrm{F}$, Teshima $\mathrm{T}$ and Ogawa $\mathrm{K}$ : Radiation dose escalation in accelerated hyperfractionated radiotherapy for stage III non-small-cell lung cancer. Anticancer Res 38: 5951-5958, 2018. PMID: 30275224. DOI: 10.21873/anticanres.12941

10 Precival C, Landy M, Poole C and Mullaney L: The role of prophylactic cranial irradiation for non-small cell lung cancer. Anticancer Res 38: 7-14, 2018. PMID: 29277750. DOI: 10.21873/anticanres. 12185

11 Saitoh JI, Shirai K, Abe T, Kubo N, Ebara T, Ohno T, Minato K, Saito R, Yamada M and Nakano T; Working Group of the Lung Tumor: A phase I study of hypofractionated carbon-ion radiotherapy for stage III non-small cell lung cancer.Anticancer Res 38: 885-891, 2018. PMID: 29374716. DOI: 10.21873/anticanres. 12298

12 Valan CD, Slagsvold JE, Halvorsen TO, Herje M, Bremnes RM, Brunsvig PF, Brustugun OT, Fløtten $\varnothing$, Levin N, Sundstrøm SH and Grønberg BH: Survival in limited disease small cell lung cancer according to N3 lymph node involvement. Anticancer Res 38: 871-876, 2018. PMID: 29374714. DOI: 10.21873/anticanres.12296

13 Horton CE, Kamal M, Leslie M, Zhang R, Tanaka T and Razaq M: Circulating tumor cells accurately predicting progressive disease after treatment in a patient with non-small cell lung cancer showing response on scans. Anticancer Res 38: 10731076, 2018. PMID: 29374743. DOI: 10.21873/anticanres. 12325

14 Zhao J, Yorke ED, Li L, Kavanagh BD, Li XA, Das S, Miften M, Rimner A, Campbell J, Xue J, Jackson A, Grimm J, Milano 
MT and Spring Kong FM: Simple factors associated with radiation-induced lung toxicity after stereotactic body radiation therapy of the thorax: A pooled analysis of 88 studies. Int $\mathrm{J}$ Radiat Oncol Biol Phys 95: 1357-1366, 2016. PMID: 27325482. DOI: 10.1016/j.ijrobp.2016.03.024

15 Dang J, Li G, Ma L, Diao R, Zang S, Han C, Zhang S and Yao $\mathrm{L}$ : Predictors of grade $\geq 2$ and grade $\geq 3$ radiation pneumonitis in patients with locally advanced non-small cell lung cancer treated with three-dimensional conformal radiotherapy. Acta Oncol 52: 1175-1180, 2013. PMID: 23198719. DOI: 10.3109/0284186X. 2012.747696

16 Palma DA, Senan S, Tsujino K, Barriger RB, Rengan R, Moreno M, Bradley JD, Kim TH, Ramella S, Marks LB, De Petris L, Stitt $\mathrm{L}$ and Rodrigues $\mathrm{G}$ : Predicting radiation pneumonitis after chemoradiation therapy for lung cancer: an international individual patient data meta-analysis. Int J Radiat Oncol Biol Phys 85: 444-450, 2013. PMID: 22682812. DOI: 10.1016/j.ijrobp.2012.04.043

17 Louvel G, Bahleda R, Ammari S, Le Péchoux C, Levy A, Massard C, Le Pavec J, Champiat S and Deutsch E: Immunotherapy and pulmonary toxicities: can concomitant immune-checkpoint inhibitors with radiotherapy increase the risk of radiation pneumonitis? Eur Respir J 51: pii:1701737, 2018 PMID: 2930192. DOI: 10.1183/13993003.01737-2017

18 Zhang XJ, Sun JG, Sun J, Ming H, Wang XX, Wu L and Chen ZT: Prediction of radiation pneumonitis in lung cancer patients: a systematic review. J Cancer Res Clin Oncol 138: 2103-2116, 2012. PMID: 22842662. DOI: 10.1007/s00432-012-1284-1

19 Kong M, Lim YJ, Kim Y, Chung MJ, Min S, Shin DO and Chung W: Diabetes mellitus is a predictive factor for radiation pneumonitis after thoracic radiotherapy in patients with lung cancer. Cancer Manag Res 11: 7103-7110, 2019. PMID: 31440097. DOI: $10.2147 / C M A R . S 210095$

20 Kalman NS, Hugo GD, Mahon RN, Deng X, Mukhopadhyay ND and Weiss E: Diabetes mellitus and radiation induced lung injury after thoracic stereotactic body radiotherapy. Radiother Oncol 129: 270-276, 2018. PMID: 30253874. DOI: 10.1016/ j.radonc.2018.08.024

21 Khan M, Siddiqui SA, Gupta MK, Seam RK and Gupta M: Normal tissue complications following hypofractionated chest wall radiotherapy in breast cancer patients and their correlation with patient, tumor, and treatment characteristics. Indian J Med Paediatr Oncol 38: 121-127, 2017. PMID: 28900318. DOI: 10.4103/ijmpo.ijmpo_80_16
22 Nalbantov G, Kietselaer B, Vandecasteele K, Oberije C, Berbee M, Troost E, Dingemans AM, van Baardwijk A, Smits K, Dekker A, Bussink J, De Ruysscher D, Lievens Y and Lambin P: Cardiac comorbidity is an independent risk factor for radiation-induced lung toxicity in lung cancer patients. Radiother Oncol 109: 100-106, 2013. PMID: 24044794. DOI: 10.1016/j.radonc.2013.08.035

23 Li F, Zhou Z, Wu A, Cai Y, Wu H, Chen M and Liang S: Preexisting radiological interstitial lung abnormalities are a risk factor for severe radiation pneumonitis in patients with smallcell lung cancer after thoracic radiation therapy. Radiat Oncol 13: 82, 2018. PMID: 29716649. DOI: 10.1186/s13014-0181030-1

24 Werner EM, Eggert MC, Bohnet S and Rades D: Prevalence and characteristics of pneumonitis following irradiation of breast cancer. Anticancer Res 39(11): 6355-6358, 2019. PMID: DOI: 10.21873/anticanres.13847

25 Shaw M, Collins BF, Ho LA and Raghu G: Rheumatoid arthritisassociated lung disease. Eur Respir Rev 24: 1-16, 2015. PMID: 25726549. DOI: 10.1183/09059180.00008014.

26 Pérez-Dórame R, Mejía M, Mateos-Toledo H and Rojas-Serrano J: Rheumatoid arthritis-associated interstitial lung disease: lung inflammation evaluated with high resolution computed tomography scan is correlated to rheumatoid arthritis disease activity. Reumatol Clin 11: 12-16, 2015. PMID: 24913966. DOI: 10.1016/j.reuma .2014 .02 .007 .

27 Wei Q, Cheng L, Hong WK and Spitz MR: Reduced DNA repair capacity in lung cancer patients. Cancer Res 56: 4103-4107, 1996. PMID: 8797573.

28 Reiter M, Baumeister P, Jaiser S, Reiss A, Schwenk-Zieger S, Kleinsasser $\mathrm{N}$ and Harréus $\mathrm{U}$ : DNA repair and mutagen sensitivity of epithelial cells and lymphocytes in oropharyngeal cancer. Oncol Lett 3: 100-106, 2012. PMID: 22740863. DOI: 10.3892/ol.2011.417

29 Sterpone S, Cornetta T, Padua L, Mastellone V, Giammarino D, Testa A, Tirindelli D, Cozzi R and Donato V: DNA repair capacity and acute radiotherapy adverse effects in Italian breast cancer patients. Mutat Res 684: 43-48, 2010. PMID: 19962393. DOI: $10.1016 /$ j.mrfmmm.2009.11.009

Received October 21, 2019

Revised October 29, 2019

Accepted October 30, 2019 